TRANSACTIONS OF THE

AMERICAN MATHEMATICAL SOCIETY

Volume 356, Number 4, Pages 1551-1568

S 0002-9947(03)03310-5

Article electronically published on October 6, 2003

\title{
STANDARD NONCOMMUTING AND COMMUTING DILATIONS OF COMMUTING TUPLES
}

\author{
B. V. RAJARAMA BHAT, TIRTHANKAR BHATTACHARYYA, AND SANTANU DEY
}

\begin{abstract}
We introduce a notion called 'maximal commuting piece' for tuples of Hilbert space operators. Given a commuting tuple of operators forming a row contraction, there are two commonly used dilations in multivariable operator theory. First there is the minimal isometric dilation consisting of isometries with orthogonal ranges, and hence it is a noncommuting tuple. There is also a commuting dilation related with a standard commuting tuple on boson Fock space. We show that this commuting dilation is the maximal commuting piece of the minimal isometric dilation. We use this result to classify all representations of the Cuntz algebra $\mathcal{O}_{n}$ coming from dilations of commuting tuples.
\end{abstract}

\section{INTRODUCTION}

It is a well-known result due to Sz.-Nagy [28] that every contraction on a Hilbert space dilates to an isometry. There is a very natural generalization of this result to a class of operator tuples defined as follows.

Definition 1. A contractive $n$-tuple, or a row contraction, is an $n$-tuple $\underline{T}=$ $\left(T_{1}, \ldots, T_{n}\right)$ of bounded operators on a Hilbert space $\mathcal{H}$ such that $T_{1} T_{1}^{*}+\cdots+$ $T_{n} T_{n}^{*} \leq I$.

Such tuples are known as row contractions, as the condition is equivalent to having the operator $\left(T_{1}, \ldots, T_{n}\right)$ from $\mathcal{H} \oplus \cdots \oplus \mathcal{H}(n$ times $)$ to $\mathcal{H}$ be a contraction. It is possible to dilate contractive tuples to tuples of isometries with orthogonal ranges. Moreover, such a dilation is unique up to unitary equivalence, under a natural minimality condition just as in the one-variable case. This dilation, which we call the minimal isometric dilation or the standard noncommuting dilation, has been explored by many authors. Some ideas along this direction can already be seen in the early paper [13] of Davis. In more concrete form this dilation can be seen in the papers of Bunce [11] and Frazho [17, [18. A very extensive study of this notion has been carried out by Popescu in a series of papers ([21]- 25], [2], [3]), and he has neat generalizations of many results from the one-variable situation. We borrow many of his ideas, particularly from his paper on the Poisson transform 24, and we also make use of the explicit structure of the 'minimal isometric dilation' he obtains in 21.

Now suppose the tuple under consideration is a commuting tuple in the sense that $T_{i} T_{j}=T_{j} T_{i}$ for all $1 \leq i, j \leq n$. Then it is natural to wish for a dilation

Received by the editors December 10, 2002 and, in revised form, February 20, 2003.

2000 Mathematics Subject Classification. Primary 47A20, 47A13, 46L05, 47D25.

Key words and phrases. Dilation, commuting tuples, complete positivity, Cuntz algebra. 
consisting of mutually commuting isometries. Unfortunately, such a dilation does not exist in general for $n \geq 3$ [20]. However, there is a dilation of commuting contractive tuples that has been studied and popularized recently by Arveson [5]. This particular dilation has also been looked at by Popescu [24], and much earlier by Drury [16] in his study of the von Neumann inequality for tuples. Similar ideas have been explored by Agler [1, Athavale [7] and others for different classes of operators using various reproducing kernels. We call this dilation of Drury, Arveson and Popescu the standard commuting dilation of contractive commuting tuples. This dilation consists of a commuting tuple, but the constituent operators are not isometries. Then a natural question arises: In what sense is this dilation canonical? In Section 3, as our main result, we show that the standard commuting dilation is the 'maximal commuting tuple contained' in the minimal isometric dilation. To begin with, we make these words inside inverted commas precise by defining what we mean by a 'maximal commuting piece' of a tuple of operators. We can explore how the standard commuting dilation of the maximal commuting piece sits inside the minimal isometric dilation of the original tuple, and whether it is the maximal commuting piece of the minimal isometric dilation, etc. We have been able to carry out this study for purely contractive tuples in Section 2.

Any tuple $\left(W_{1}, \ldots, W_{n}\right)$ of isometries with orthogonal ranges satisfying $\sum W_{i} W_{i}^{*}$ $=I$ gives us a concrete representation of the familiar Cuntz algebra [12]. Recently, there has been a lot of effort to study such representations in connection with wavelet theory, see for instance the papers [9], [10] of Bratteli and Jorgensen. If we start with a contractive tuple $\left(T_{1}, \ldots, T_{n}\right)$ satisfying $\sum T_{i} T_{i}^{*}=I$ and consider the minimal isometric dilation, we actually have a representation of the Cuntz algebra. This was proved by Popescu in [21]. Very interesting results on classification of these representations up to unitary equivalence in terms of invariants determined by $\left(T_{1}, \ldots, T_{n}\right)$ have been obtained by Davidson, Kribs, and Shpigel [14], where the operators $T_{i}$ act on a finite dimensional space. It is natural to ask what representations of the Cuntz algebra can one get by dilating contractive tuples which are also commuting. Surprisingly, they are very few and are all determined by the GNS representations of the so-called Cuntz states. This result we obtain in Section 4 , as an application of the main result. Unlike the work of Davidson et al., we need not restrict the operators $T_{i}$ to be acting on finite dimensional spaces.

All the Hilbert spaces we consider will be complex and separable. For a subspace $\mathcal{H}$ of a Hilbert space, $P_{\mathcal{H}}$ will denote the orthogonal projection onto $\mathcal{H}$. For fixed $n \geq 2$, we need two standard $n$-tuples of operators, denoted by $\underline{V}$ and $\underline{S}$, acting on Fock spaces. For any Hilbert space $\mathcal{K}$, we have the full Fock space over $\mathcal{K}$ denoted by $\Gamma(\mathcal{K})$ and the boson (or symmetric) Fock space over $\mathcal{K}$ denoted by $\Gamma_{s}(\mathcal{K})$, defined as

$$
\begin{aligned}
\Gamma(\mathcal{K}) & =\mathbb{C} \oplus \mathcal{K} \oplus \mathcal{K}^{\otimes^{2}} \oplus \cdots \oplus \mathcal{K}^{\otimes^{m}} \oplus \cdots \\
\Gamma_{s}(\mathcal{K}) & =\mathbb{C} \oplus \mathcal{K} \oplus \mathcal{K}^{\Im^{2}} \oplus \cdots \oplus \mathcal{K}^{\Im^{m}} \oplus \cdots
\end{aligned}
$$

where $\mathcal{K}^{\Im^{m}}$ denotes the $m$-fold symmetric tensor product. We will consider the boson Fock space as a subspace of the full Fock space in the natural way. We denote the vacuum vector $1 \oplus 0 \oplus \cdots$ (in either of these Fock spaces) by $\omega$. Let $\mathbb{C}^{n}$ be the $n$-dimensional complex Euclidian space with the usual inner product, and $\Gamma\left(\mathbb{C}^{n}\right)$ the full Fock space over $\mathbb{C}^{n}$. Let $\left\{e_{1}, \ldots, e_{n}\right\}$ be the standard orthonormal 
basis of $\mathbb{C}^{n}$. Then the (left) creation operators $V_{i}$ on $\Gamma\left(\mathbb{C}^{n}\right)$ are defined by

$$
V_{i} x=e_{i} \otimes x,
$$

where $1 \leq i \leq n$ and $x \in \Gamma\left(\mathbb{C}^{n}\right)$ (of course, here $e_{i} \otimes \omega$ is interpreted as $e_{i}$ ). It is obvious that the tuple $\underline{V}=\left(V_{1}, \ldots, V_{n}\right)$ consists of isometries with orthogonal ranges, and it is contractive; in fact, $\sum V_{i} V_{i}^{*}=I-E_{0}$, where $E_{0}$ is the projection onto the vacuum space. Let $\underline{S}=\left(S_{1}, \ldots, S_{n}\right)$ be the tuple of operators on $\Gamma_{s}\left(\mathbb{C}^{n}\right)$, where $S_{i}$ is the compression of $V_{i}$ to $\Gamma_{s}\left(\mathbb{C}^{n}\right)$ :

$$
S_{i}=\left.P_{\Gamma_{s}\left(\mathbb{C}^{n}\right)} V_{i}\right|_{\Gamma_{s}\left(\mathbb{C}^{n}\right)} .
$$

Clearly each $V_{i}^{*}$ leaves $\Gamma_{s}\left(\mathbb{C}^{n}\right)$ invariant. Therefore, $S_{i}^{*} x=V_{i}^{*} x$, for $x \in \Gamma_{s}\left(\mathbb{C}^{n}\right)$. Then it is easy to see that $\left(S_{1}, \ldots, S_{n}\right)$ is also a contractive tuple satisfying $\sum S_{i} S_{i}^{*}$ $=I^{s}-E_{0}^{s}$ (where now $I^{s}, E_{0}^{s}$ are respectively the identity and the projection onto the vacuum space in $\Gamma_{s}\left(\mathbb{C}^{n}\right)$ ). Moreover, a simple computation shows that $S_{i} S_{j}=S_{j} S_{i}$ for all $1 \leq i, j \leq n$.

For operator tuples $\left(T_{1}, \ldots, T_{n}\right)$, quite often we need to consider products of the form $T_{\alpha_{1}} T_{\alpha_{2}} \ldots T_{\alpha_{m}}$, where each $\alpha_{k} \in\{1,2, \ldots, n\}$. So it is convenient to have a notation for such products. Let $\Lambda$ denote the set $\{1,2, \ldots, n\}$ and $\Lambda^{m}$ denote the $m$-fold cartesian product of $\Lambda$ for $m \geq 1$. Given $\alpha=\left(\alpha_{1}, \ldots, \alpha_{m}\right)$ in $\Lambda^{m}, \underline{T}^{\alpha}$ will mean the operator $T_{\alpha_{1}} T_{\alpha_{2}} \cdots T_{\alpha_{m}}$. Let $\tilde{\Lambda}$ denote $\bigcup_{n=0}^{\infty} \Lambda^{n}$, where $\Lambda^{0}$ is just the set $\{0\}$ by convention, and by $\underline{T}^{0}$ we would mean the identity operator of the Hilbert space where the operators $T_{i}$ are acting. In a similar fashion, for $\alpha \in \tilde{\Lambda}, e^{\alpha}$ will denote the vector $e_{\alpha_{1}} \otimes e_{\alpha_{2}} \otimes \cdots \otimes e_{\alpha_{m}}$ in the full Fock space $\Gamma\left(\mathbb{C}^{n}\right)$, and $e^{0}$ is the vacuum $\omega$.

\section{Maximal COMmuting Piece AND Dilation}

Definition 2. Let $\mathcal{H}, \mathcal{L}$ be two Hilbert spaces such that $\mathcal{H}$ is a closed subspace of $\mathcal{L}$. Suppose $\underline{T}, \underline{R}$ are $n$-tuples of bounded operators on $\mathcal{H}, \mathcal{L}$ respectively. Then $\underline{R}$ is called a dilation of $\underline{T}$ if

$$
R_{i}^{*} u=T_{i}^{*} u
$$

for all $u \in \mathcal{H}, 1 \leq i \leq n$. In such a case $\underline{T}$ is called a piece of $\underline{R}$. If further $\underline{T}$ is a commuting tuple (i.e., $T_{i} T_{j}=T_{j} T_{i}$ for all $i, j$ ), then it is called a commuting piece of $\underline{R}$. A dilation $\underline{R}$ of $\underline{T}$ is said to be a minimal dilation if $\overline{\operatorname{span}}\left\{\underline{R}^{\alpha} h: \alpha \in \tilde{\Lambda}, h \in\right.$ $\mathcal{H}\}=\mathcal{L}$.

In this definition we note that if $\underline{R}$ is a dilation of $\underline{T}$, then $\mathcal{H}$ is a co-invariant subspace of $\underline{R}$, that is, all $R_{i}^{*}$ leave it invariant. It is standard (see [19]) to call $\left(R_{1}^{*}, \ldots, R_{n}^{*}\right)$ an extension of $\left(T_{1}^{*}, \ldots, T_{n}^{*}\right)$ and $\left(T_{1}^{*}, \ldots, T_{n}^{*}\right)$ a part of $\left(R_{1}^{*}, \ldots, R_{n}^{*}\right)$. In such a situation it is easy to see that for any $\alpha, \beta \in \tilde{\Lambda}, \underline{T}^{\alpha}\left(\underline{T}^{\beta}\right)^{*}$ is the compression of $\underline{R}^{\alpha}\left(\underline{R}^{\beta}\right)^{*}$ to $\mathcal{H}$, that is,

$$
\underline{T}^{\alpha}\left(\underline{T}^{\beta}\right)^{*}=\left.P_{\mathcal{H}} \underline{R}^{\alpha}\left(\underline{R}^{\beta}\right)^{*}\right|_{\mathcal{H}} .
$$

We may extend this relation to any polynomials $p, q$ in $n$ noncommuting variables, to get

$$
p(\underline{T})(q(\underline{T}))^{*}=\left.P_{\mathcal{H}} p(\underline{R})(q(\underline{R}))^{*}\right|_{\mathcal{H}} .
$$

Usually property (2.1) is all that one demands of a dilation. But we have imposed a condition of co-invariance in Definition 2, as it is very convenient to have it this way for our purposes. 
Now we look at commuting pieces of tuples. Let $\underline{R}$ be a $n$-tuple of bounded operators on a Hilbert space $\mathcal{L}$. Consider

$$
\mathcal{C}(\underline{R})=\left\{\mathcal{M}: \mathcal{M} \text { is a co-invariant subspace for each } R_{i},\right.
$$

$$
\left.R_{i}^{*} R_{j}^{*} h=R_{j}^{*} R_{i}^{*} h, \forall h \in \mathcal{M}, \forall i, j\right\} .
$$

So $\mathcal{C}(\underline{R})$ consists of all co-invariant subspaces of an $n$-tuple of operators $\underline{R}$ such that the compressions form a commuting tuple. It is a complete lattice, in the sense that arbitrary intersections and span closures of arbitrary unions of such spaces are again in this collection. Therefore it has a maximal element. We denote it by $\mathcal{L}^{c}(\underline{R})$ (or by $\mathcal{L}^{c}$ when the tuple under consideration is clear).

Definition 3. Suppose $\underline{R}=\left(R_{1}, \cdots, R_{n}\right)$ is an $n$-tuple of operators on a Hilbert space $\mathcal{L}$. Then the maximal commuting piece of $\underline{R}$ is defined as the commuting piece $\underline{R}^{c}=\left(R_{1}^{c}, \ldots, R_{n}^{c}\right)$ obtained by compressing $\underline{R}$ to the maximal element $\mathcal{L}^{c}(\underline{R})$ of $\mathcal{C}(\underline{R})$. The maximal commuting piece is said to be trivial if the space $\mathcal{L}^{c}(\underline{R})$ is just the zero space.

It is quite easy to get tuples with trivial commuting piece, as tuples with no nontrivial co-invariant subspaces have this property. Of course, our main interest lies in tuples with nontrivial commuting pieces. The following result is quite useful in determining the maximal commuting piece.

Proposition 4. Let $\underline{R}$ be an n-tuple of bounded operators on a Hilbert space $\mathcal{L}$. Let $\mathcal{K}_{i j}=\overline{\operatorname{span}}\left\{\underline{R}^{\alpha}\left(R_{i} R_{j}-R_{j} R_{i}\right) h: h \in \mathcal{L}, \alpha \in \tilde{\Lambda}\right\}$ for all $1 \leq i, j \leq n$, and $\mathcal{K}=\overline{\operatorname{span}}\left\{\bigcup_{i, j=1}^{n} \mathcal{K}_{i j}\right\}$. Then $\mathcal{L}^{c}(\underline{R})=\mathcal{K}^{\perp}$. In other words, $\mathcal{L}^{c}(\underline{R})=\{h \in \mathcal{L}:$ $\left.\left(R_{i}^{*} R_{j}^{*}-R_{j}^{*} R_{i}^{*}\right)\left(\underline{R}^{\alpha}\right)^{*} h=0, \forall 1 \leq i, j \leq n, \alpha \in \tilde{\Lambda}\right\}$.

Proof. First, $\mathcal{K}^{\perp}$ is obviously a co-invariant subspace of $\underline{R}$, as each $R_{i}$ leaves $\mathcal{K}$ invariant. Now for $i, j \in\{1,2, \ldots, n\}$, and $h_{1} \in \mathcal{L}^{c}, h_{2} \in \mathcal{L}$,

$$
\left\langle\left(R_{i}^{*} R_{j}^{*}-R_{j}^{*} R_{i}^{*}\right) h_{1}, h_{2}\right\rangle=\left\langle h_{1},\left(R_{j} R_{i}-R_{i} R_{j}\right) h_{2}\right\rangle=0 .
$$

So we get $\left(R_{i}^{*} R_{j}^{*}-R_{j}^{*} R_{i}^{*}\right) h_{1}=0$. Now if $\mathcal{M}$ is an element of $\mathcal{C}(\underline{R})$, take $i, j \in$ $\{1, \ldots, n\}, \alpha \in \tilde{\Lambda}, h_{1} \in \mathcal{M}, h \in \mathcal{L}$. We have

$$
\left\langle h_{1}, \underline{R}^{\alpha}\left(R_{i} R_{j}-R_{j} R_{i}\right) h\right\rangle=\left\langle\left(R_{j}^{*} R_{i}^{*}-R_{i}^{*} R_{j}^{*}\right)\left(\underline{R}^{\alpha}\right)^{*} h_{1}, h\right\rangle=0,
$$

as $\left(\underline{R}^{\alpha}\right)^{*} h_{1} \in \mathcal{M}$. Hence $\mathcal{M}$ is contained in $\mathcal{K}^{\perp}$. Now the last statement is easy to see.

Corollary 5. Suppose $\underline{R}, \underline{T}$ are $n$-tuples of operators on two Hilbert spaces $\mathcal{L}, \mathcal{M}$. Then the maximal commuting piece of $\left(R_{1} \oplus T_{1}, \ldots, R_{n} \oplus T_{n}\right)$ acting on $\mathcal{L} \oplus \mathcal{M}$ is $\left(R_{1}^{c} \oplus T_{1}^{c}, \ldots, R_{n}^{c} \oplus T_{n}^{c}\right)$ acting on $\mathcal{L}^{c} \oplus \mathcal{M}^{c}$. The maximal commuting piece of $\left(R_{1} \otimes I, \ldots, R_{n} \otimes I\right)$ acting on $\mathcal{L} \otimes \mathcal{M}$ is $\left(R_{1}^{c} \otimes I, \ldots, R_{n}^{c} \otimes I\right)$ acting on $\mathcal{L}^{c} \otimes \mathcal{M}$.

Proof. Clear from Proposition 4.

Proposition 6. Let $\underline{V}=\left(V_{1}, \ldots, V_{n}\right)$ and $\underline{S}=\left(S_{1}, \ldots, S_{n}\right)$ be standard contractive tuples on the full Fock space $\Gamma\left(\mathbb{C}^{n}\right)$ and the boson Fock space $\Gamma_{s}\left(\mathbb{C}^{n}\right)$ respectively. Then the maximal commuting piece of $\underline{V}$ is $\underline{S}$.

Proof. As we have already noted in the Introduction, $\underline{S}$ is a commuting piece of $\underline{V}$. To show maximality we make use of Proposition 4. Suppose $x \in \Gamma\left(\mathbb{C}^{n}\right)$ and $\left\langle x, \underline{V}^{\alpha}\left(V_{i} V_{j}-V_{j} V_{i}\right) y\right\rangle=0$ for all $\alpha \in \tilde{\Lambda}, 1 \leq i, j \leq n$ and $y \in \Gamma\left(\mathbb{C}^{n}\right)$. We wish 
to show that $x \in \Gamma_{s}\left(\mathbb{C}^{n}\right)$. Suppose $x_{m}$ is the $m$-particle component of $x$, that is, $x=\bigoplus_{m>0} x_{m}$ with $x_{m} \in\left(\mathbb{C}^{n}\right)^{\otimes^{m}}$ for $m \geq 0$. For $m \geq 2$ and any permutation $\sigma$ of $\{1,2, \ldots, m\}$ we need to show that the unitary $U_{\sigma}:\left(\mathbb{C}^{n}\right)^{\otimes^{m}} \rightarrow\left(\mathbb{C}^{n}\right)^{\otimes^{m}}$, defined by

$$
U_{\sigma}\left(u_{1} \otimes \cdots \otimes u_{m}\right)=u_{\sigma^{-1}(1)} \otimes \cdots \otimes u_{\sigma^{-1}(m)},
$$

leaves $x_{m}$ fixed. Since the group of permutations of $\{1,2, \ldots, m\}$ is generated by permutations $\{(1,2), \ldots,(m-1, m)\}$ it is enough to verify $U_{\sigma}\left(x_{m}\right)=x_{m}$ for permutations $\sigma$ of the form $(i, i+1)$. So fix $m$ and $i$ with $m \geq 2$ and $1 \leq i \leq(m-1)$. We have

$$
\left\langle\bigoplus_{p} x_{p}, \underline{V}^{\alpha}\left(V_{k} V_{l}-V_{l} V_{k}\right) y\right\rangle=0
$$

for every $y \in \Gamma\left(\mathbb{C}^{n}\right), 1 \leq k, l \leq n$. As $\alpha$ is arbitrary, this means that

$$
\left\langle x_{m}, z \otimes\left(e_{k} \otimes e_{l}-e_{l} \otimes e_{k}\right) \otimes w\right\rangle=0
$$

for any $z \in\left(\mathbb{C}^{n}\right)^{\otimes^{(i-1)}}, w \in\left(\mathbb{C}^{n}\right)^{\otimes^{(m-i-1)}}$. This clearly implies $U_{\sigma}\left(x_{m}\right)=x_{m}$, for $\sigma=(i, i+1)$.

Now let us see how the maximal commuting piece behaves with respect to the operation of taking dilations. Before considering specific dilations, we have the following general statement.

Proposition 7. Suppose $\underline{T}, \underline{R}$ are $n$-tuples of bounded operators on $\mathcal{H}, \mathcal{L}$, with $\mathcal{H} \subseteq \mathcal{L}$, such that $\underline{R}$ is a dilation of $\underline{T}$. Then $\mathcal{H}^{c}(\underline{T})=\mathcal{L}^{c}(\underline{R}) \cap \mathcal{H}$ and $\underline{R}^{c}$ is a dilation of $\underline{T}^{c}$.

Proof. We have $R_{i}^{*} h=T_{i}^{*} h$, for $h \in \mathcal{H}$. Therefore, $\left(R_{i}^{*} R_{j}^{*}-R_{j}^{*} R_{i}^{*}\right)\left(\underline{R}^{\alpha}\right)^{*} h=$ $\left(T_{i}^{*} T_{j}^{*}-T_{j}^{*} T_{i}^{*}\right)\left(\underline{T}^{\alpha}\right)^{*} h$ for $h \in \mathcal{H}, 1 \leq i, j \leq n$, and $\alpha \in \tilde{\Lambda}$. Now the first part of the result is clear from Proposition 4. Further, for $h \in \mathcal{L}^{c}(\underline{R})$ we have $R_{i}^{*} h=\left(R_{i}^{c}\right)^{*} h$, and so for $h \in \mathcal{H}^{c}(\underline{T})=\mathcal{L}^{c}(\underline{R}) \cap \mathcal{H}$ we have $\left(R_{i}^{c}\right)^{*} h=R_{i}^{*} h=T_{i}^{*} h=\left(T_{i}^{c}\right)^{*} h$. This proves the claim.

Definition 8. Let $\underline{T}=\left(T_{1}, \ldots, T_{n}\right)$ be a contractive tuple on a Hilbert space $\mathcal{H}$. The operator $\Delta_{\underline{T}}=\left[I-\left(T_{1} T_{1}^{*}+\cdots+T_{n} T_{n}^{*}\right)\right]^{\frac{1}{2}}$ is called the defect operator of $\underline{T}$ and the subspace $\overline{\Delta_{T}(\mathcal{H})}$ is called the defect space of $\underline{T}$. The tuple $\underline{T}$ is said to be pure if $\left.\sum_{\alpha \in \Lambda^{m}} \underline{T}^{\alpha} \underline{T}^{\alpha}\right)^{*}$ converges to zero in the strong operator topology as $m$ tends to infinity.

Suppose $\sum T_{i} T_{i}^{*}=I$; then it is easy to see that $\sum_{\alpha \in \Lambda^{m}} \underline{T}^{\alpha}\left(\underline{T}^{\alpha}\right)^{*}=I$ for all $m$, and there is no way this sequence can converge to zero. So in the pure case the defect operator and the defect spaces are nontrivial.

First we restrict our attention to pure tuples. The reason for this is that it is very easy to write down standard dilations for pure tuples. So let $\mathcal{H}$ be a complex, separable Hilbert space and let $\underline{T}$ be a pure contractive tuple on $\mathcal{H}$. Take $\tilde{\mathcal{H}}=$ $\Gamma\left(\mathbb{C}^{n}\right) \otimes \overline{\Delta_{\underline{T}}(\mathcal{H})}$, and define an operator $A: \mathcal{H} \rightarrow \tilde{\mathcal{H}}$ by

$$
A h=\sum_{\alpha} e^{\alpha} \otimes \Delta_{\underline{T}}\left(\underline{T}^{\alpha}\right)^{*} h,
$$

where the sum is taken over all $\alpha \in \tilde{\Lambda}$. It is well-known ([24], [2]), and also easily verifiable using the pureness of $\underline{T}$, that $A$ is an isometry with

$$
A^{*}\left(e^{\alpha} \otimes h\right)=\underline{T}^{\alpha} \Delta_{\underline{T}} h \quad \text { for } \alpha \in \tilde{\Lambda}, h \in \overline{\Delta_{\underline{T}}(\mathcal{H})} .
$$


Now $\mathcal{H}$ is considered as a subspace of $\tilde{\mathcal{H}}$ by identifying vectors $h \in \mathcal{H}$ with $A h \in$ $\tilde{\mathcal{H}}$. Then by noting that each $V_{i}^{*} \otimes I$ leaves the range of $A$ invariant and $\underline{T}^{\alpha}=$ $A^{*}\left(\underline{V}^{\alpha} \otimes I\right) A$ for all $\alpha \in \tilde{\Lambda}$, it is seen that the tuple $\underline{\tilde{V}}=\left(V_{1} \otimes I, \ldots, V_{n} \otimes I\right)$ of operators on $\tilde{\mathcal{H}}$ is a realization of the minimal isometric dilation of $\underline{T}$. Now if $\underline{T}$ is a commuting tuple, it is easy to see that the range of $A$ is contained in $\tilde{\mathcal{H}}_{s}=$ $\Gamma_{s}\left(\mathbb{C}^{n}\right) \otimes \overline{\Delta_{\underline{T}}(\mathcal{H})}$. In other words, now $\mathcal{H}$ can be considered as a subspace of $\tilde{\mathcal{H}}_{s}$. Moreover, $\underline{\underline{S}}=\left(S_{1} \otimes I, \ldots, S_{n} \otimes I\right)$, as a tuple of operators in $\tilde{\mathcal{H}}_{s}$ is a realization of the standard commuting dilation of $\left(T_{1}, \ldots, T_{n}\right)$. More abstractly, if $\underline{T}$ is commuting and pure, the standard commuting dilation of it is got by embedding $\mathcal{H}$ isometrically in $\Gamma_{s}\left(\mathbb{C}^{n}\right) \otimes \mathcal{K}$, for some Hilbert space $\mathcal{K}$, such that $\left(S_{1} \otimes I_{\mathcal{K}}, \ldots, S_{n} \otimes I_{\mathcal{K}}\right)$ is a dilation of $\underline{T}$ and $\overline{\operatorname{span}}\left\{\left(\underline{S}^{\alpha} \otimes I_{\mathcal{K}}\right) h: h \in \mathcal{H}, \alpha \in \tilde{\Lambda}\right\}=\Gamma_{s}\left(\mathbb{C}^{n}\right) \otimes \mathcal{K}$. Up to unitary equivalence there is a unique such dilation, and $\operatorname{dim}(\mathcal{K})=\operatorname{rank}\left(\Delta_{\underline{T}}\right)$.

Theorem 9. Let $\underline{T}$ be a pure contractive tuple on a Hilbert space $\mathcal{H}$. Then the maximal commuting piece $\tilde{V}^{c}$ of the minimal isometric dilation $\underline{\tilde{V}}$ of $\underline{T}$ is a realization of the standard commuting dilation of $\underline{T}^{c}$ if and only if $\overline{\Delta_{\underline{T}}(\mathcal{H})}=\overline{\Delta_{\underline{T}}\left(\mathcal{H}^{c}(\underline{T})\right)}$. In such a case $\operatorname{rank}\left(\Delta_{\underline{T}}\right)=\operatorname{rank}\left(\Delta_{\underline{T}^{c}}\right)=\operatorname{rank}\left(\Delta_{\underline{\tilde{V}}}\right)=\operatorname{rank}\left(\Delta_{\underline{\tilde{V}}^{c}}\right)$.

Proof. We denote $\mathcal{H}^{c}(\underline{T}), \overline{\Delta_{\underline{T}}(\mathcal{H})}$ and $\overline{\Delta_{\underline{T}}\left(\mathcal{H}^{c}(\underline{T})\right)}$ by $\mathcal{H}^{c}, \mathcal{M}$, and $\mathcal{M}^{c}$ respectively. It is obvious that $\underline{T}^{c}$ is also a pure contractive tuple. We already know from Proposition 7 that $\underline{\tilde{V}}^{c}=\left(\underline{S} \otimes I_{\mathcal{M}}\right)$ on $\Gamma_{s}\left(\mathbb{C}^{n}\right) \otimes \mathcal{M}$ is a dilation of $\underline{T}^{c}$. It is the standard dilation if and only if $\mathcal{L}:=\overline{\operatorname{span}}\left\{\left(\underline{S}^{\alpha} \otimes I_{\mathcal{M}}\right) A h: h \in \mathcal{H}^{c}, \alpha \in \tilde{\Lambda}\right\}$ is equal to $\Gamma_{s}\left(\mathbb{C}^{n}\right) \otimes \mathcal{M}$, where $A: \mathcal{H} \rightarrow \tilde{\mathcal{H}}$ is the isometry defined by (2.2).

From the definition of $A$, using the commutativity of the operators $T_{i}$, it is clear that for $h \in \mathcal{H}^{c}, A h \in \Gamma_{s}\left(\mathbb{C}^{n}\right) \otimes \mathcal{M}^{c}$. Hence $\mathcal{L} \subseteq \Gamma_{s}\left(\mathbb{C}^{n}\right) \otimes \mathcal{M}^{c}$. Further, as $\left(\underline{S} \otimes I_{\mathcal{M}}\right)$ is a dilation, $\left(S_{i}^{*} \otimes I_{\mathcal{M}}\right)$ leaves $A\left(\mathcal{H}^{c}\right)$ invariant. Therefore, $\left(\left(I-\sum S_{i} S_{i}^{*}\right) \otimes I_{\mathcal{M}}\right) A h \in$ $\mathcal{L}$ for $h \in \mathcal{H}^{c}$. But, $\left(I-\sum S_{i} S_{i}^{*}\right)$ being the projection onto the vacuum space, $\left(\left(I-\sum S_{i} S_{i}^{*}\right) \otimes I_{\mathcal{M}}\right) A h=\omega \otimes \Delta_{\underline{T}} h$. As $\left\{\underline{S}^{\alpha} \omega, \alpha \in \tilde{\Lambda}\right\}$ spans whole of $\Gamma_{s}\left(\mathbb{C}^{n}\right)$, we get that $\Gamma_{s}\left(\mathbb{C}^{n}\right) \otimes \mathcal{M}^{c} \subseteq \mathcal{L}$. Hence $\mathcal{L}=\Gamma_{s}\left(\mathbb{C}^{n}\right) \otimes \mathcal{M}^{c}$, and we have proved the first claim.

Now suppose $\tilde{V}^{c}$ is a realization of the standard commuting dilation of $\underline{T}^{c}$. This in particular means that $\operatorname{rank}\left(\Delta_{\underline{T}^{c}}\right)=\operatorname{rank}\left(\Delta_{\tilde{V}^{c}}\right)$. Also as $\underline{\tilde{V}}$ is the minimal isometric dilation of $\underline{T}, \operatorname{rank}\left(\Delta_{\underline{T}}\right)=\operatorname{rank}\left(\Delta_{\tilde{\underline{V}}}\right)$. Further as $\underline{\tilde{V}}^{c}=\left(\underline{S} \otimes I_{\mathcal{M}}\right)$, $\operatorname{rank}\left(\Delta_{\tilde{V}^{c}}\right)=\operatorname{dim}(\mathcal{M})=\operatorname{rank}\left(\Delta_{\underline{T}}\right)$.

We may ask whether the equality of ranks in this theorem is good enough to make a converse statement. To answer this we make use of the following simple lemma.

Lemma 10. Suppose

$$
M=\left[\begin{array}{cc}
A & B^{*} \\
B & C
\end{array}\right]
$$

is a bounded positive operator on some Hilbert space. Then

$$
\operatorname{rank}(A)=\operatorname{rank}\left(\left[\begin{array}{c}
A \\
B
\end{array}\right]\right)
$$

Proof. Without loss of generality we can assume that $M$ is a contraction. Then it is a folklore theorem that there exists a contraction $D$ such that $B=C^{\frac{1}{2}} D A^{\frac{1}{2}}$. 
Now

$$
\left[\begin{array}{l}
A \\
B
\end{array}\right]=\left[\begin{array}{c}
A^{\frac{1}{2}} \\
C^{\frac{1}{2}} D
\end{array}\right]\left[A^{\frac{1}{2}}\right]
$$

and hence $\operatorname{rank}\left(\left[{ }_{B}^{A}\right]\right) \leq \operatorname{rank}\left(A^{\frac{1}{2}}\right)$. But $A$ being positive, $\operatorname{rank}(A)=\operatorname{rank}\left(A^{\frac{1}{2}}\right)$. Therefore $\operatorname{rank}\left(\left[\begin{array}{c}A \\ B\end{array}\right]\right) \leq \operatorname{rank}(A) \leq \operatorname{rank}\left(\left[\begin{array}{c}A \\ B\end{array}\right]\right)$.

Remark 11. Let $\underline{T}$ be a pure contractive tuple on a Hilbert space $\mathcal{H}$ with minimal isometric dilation $\underline{\underline{V}}$. If rank $\Delta_{\underline{T}}$ and $\operatorname{rank} \Delta_{\underline{T}^{c}}$ are finite and equal, then $\tilde{V}^{c}$ is a realization of the standard commuting dilation of $\underline{T}^{c}$.

Proof. In view of Theorem 9 we need to show that $\overline{\Delta_{\underline{T}}(\mathcal{H})}=\overline{\Delta_{\underline{T}}\left(\mathcal{H}^{c}(\underline{T})\right)}$. Since $\overline{\Delta_{\underline{T}}(\mathcal{H})} \supseteq \overline{\Delta_{\underline{T}}\left(\mathcal{H}^{c}(\underline{T})\right)}$, and these spaces are now finite dimensional, it suffices to show that their dimensions are equal, or $\operatorname{rank}\left(\Delta_{\underline{T}}\right)=\operatorname{rank}\left(\Delta_{\underline{T}} P_{\mathcal{H}^{c}}\right)$. Clearly $\operatorname{rank}\left(\Delta_{\underline{T}}\right) \geq \operatorname{rank}\left(\Delta_{\underline{T}} P_{\mathcal{H}^{c}}\right)$. Also by assumption, $\operatorname{rank}\left(\Delta_{\underline{T}}\right)=\operatorname{rank}\left(\Delta_{\underline{T}^{c}}\right)$. By positivity $\operatorname{rank}\left(\Delta_{\underline{T}^{c}}\right)=\operatorname{rank}\left(\Delta_{T^{c}}^{2}\right)$. And then by the previous lemma $\operatorname{rank}\left(\Delta_{\underline{T}^{c}}^{2}\right)=$ $\operatorname{rank}\left(P_{\mathcal{H}^{c}}\left(\Delta_{\underline{T}}^{2}\right) P_{\mathcal{H}^{c}}\right)=\operatorname{rank}\left(\overline{\Delta_{T}^{2}} P_{\mathcal{H}^{c}}\right) \leq \operatorname{rank}\left(\Delta_{\underline{T}} P_{\mathcal{H}^{c}}\right)$.

If both the ranks are infinite, we cannot ensure that $\overline{\Delta_{\underline{T}}(\mathcal{H})}=\overline{\Delta_{\underline{T}}\left(\mathcal{H}^{c}(\underline{T})\right)}$, as seen by the following example.

Example 12. Let $\underline{R}=\left(R_{1}, R_{2}\right)$ be a commuting pure contractive 2-tuple on an infinite dimensional Hilbert space $\mathcal{H}_{0}$ (We can even take $R_{1}, R_{2}$ as scalars) such that $\overline{\Delta_{\underline{R}}\left(\mathcal{H}_{0}\right)}$ is infinite dimensional. Take $\mathcal{H}=\mathcal{H}_{0} \oplus \mathbb{C}^{2}$, and let $T_{1}, T_{2}$ be operators on $\mathcal{H}$ defined by

$$
T_{1}=\left[\begin{array}{ccc}
R_{1} & & \\
& 0 & t_{1} \\
& 0 & 0
\end{array}\right], \quad T_{2}=\left[\begin{array}{ccc}
R_{2} & & \\
& 0 & 0 \\
& t_{2} & 0
\end{array}\right],
$$

where $t_{1}, t_{2}$ are any two scalars, $0<t_{1}, t_{2}<1$. Then $\underline{T}=\left(T_{1}, T_{2}\right)$ is a pure contractive tuple. Making use of Corollary $5, \mathcal{H}^{c}(\underline{T})=\mathcal{H}_{0}$ (thought of as a subspace of $\mathcal{H}$ in the natural way) and the maximal commuting piece of $\underline{T}$ is $\left(R_{1}, R_{2}\right)$, and therefore $\operatorname{rank}\left(\Delta_{\underline{T}^{c}}\right)=\operatorname{rank}\left(\Delta_{\underline{T}}\right)=\infty$. But $\overline{\Delta_{\underline{T}}(\mathcal{H})}=\overline{\Delta_{\underline{R}}\left(\mathcal{H}_{0}\right)} \oplus \mathbb{C}^{2}$.

We do not know how to extend Theorem 9 to contractive tuples which are not necessarily pure.

\section{Commuting tuples}

In this section we wish to consider commutative contractive tuples. Let us begin by describing how one obtains two standard dilations for such tuples.

Recall standard tuples $\underline{V}$ and $\underline{S}$ on Fock spaces $\Gamma\left(\mathbb{C}^{n}\right)$ and $\Gamma_{s}\left(\mathbb{C}^{n}\right)$, respectively, introduced in the Introduction. Let $C^{*}(\underline{V})$ and $C^{*}(\underline{S})$ be unital $C^{*}$ algebras generated by them. For any $\alpha, \beta \in \tilde{\Lambda}, \underline{V}^{\alpha}\left(I-\sum V_{i} V_{i}^{*}\right)\left(\underline{\underline{S}}^{\beta}\right)^{*}$ is the rank one operator $x \mapsto\left\langle e^{\beta}, x\right\rangle e^{\alpha}$ formed by basis vectors $e^{\alpha}, e^{\beta}$. So $C^{*}(\underline{V})$ contains all compact operators. In a similar way we see that $C^{*}(\underline{S})$ also contains all compact operators of $\Gamma_{s}\left(\mathbb{C}^{n}\right)$. As $V_{i}^{*} V_{j}=\delta_{i j} I$, it is easy to see that $C^{*}(\underline{V})=\overline{\operatorname{span}}\left\{\underline{V}^{\alpha}\left(\underline{V}^{\beta}\right)^{*}\right.$ : $\alpha, \beta \in \tilde{\Lambda}\}$. By explicit computation we see that the commutators $\left[S_{i}^{*}, S_{j}\right]$ are compact for all $i, j$ (see [5], Proposition 5.3, or [8]). Therefore we can also obtain $C^{*}(\underline{S})=\overline{\operatorname{span}}\left\{\underline{S}^{\alpha}\left(\underline{S}^{\beta}\right)^{*}: \alpha, \beta \in \tilde{\Lambda}\right\}$.

Suppose $\underline{T}$ is a contractive tuple on a Hilbert space $\mathcal{H}$. We obtain a certain completely positive map (Popescu's Poisson transform) from $C^{*}(\underline{V})$ to $\mathcal{B}(\mathcal{H})$, as 
follows. For $0<r<1$ the tuple $r \underline{T}=\left(r T_{1}, \ldots, r T_{n}\right)$ is clearly a pure contraction. So by $(2.2)$ we have an isometry $A_{r}: \mathcal{H} \rightarrow \Gamma\left(\mathbb{C}^{n}\right) \otimes \overline{\Delta_{r}(\mathcal{H})}$ defined by

$$
A_{r} h=\sum_{\alpha} e^{\alpha} \otimes \Delta_{r}\left((r \underline{T})^{\alpha}\right)^{*} h, h \in \mathcal{H},
$$

where $\Delta_{r}=\left(I-r^{2} \sum T_{i} T_{i}^{*}\right)^{\frac{1}{2}}$. So for every $0<r<1$ we have a completely positive $\operatorname{map} \psi_{r}: C^{*}(\underline{V}) \rightarrow \mathcal{B}(\mathcal{H})$ defined by

$$
\psi_{r}(X)=A_{r}^{*}(X \otimes I) A_{r}, X \in C^{*}(\underline{V}) .
$$

By taking the limit as $r$ increases to 1 (see 24] or 2] for details), we obtain a unital completely positive map $\psi$ from $C^{*}(\underline{V})$ to $\mathcal{B}(\mathcal{H})$ satisfying

$$
\psi\left(\underline{V}^{\alpha}\left(\underline{V}^{\beta}\right)^{*}\right)=\underline{T}^{\alpha}\left(\underline{T}^{\beta}\right)^{*} \text { for } \alpha, \beta \in \tilde{\Lambda} .
$$

As $C^{*}(\underline{V})=\overline{\operatorname{span}}\left\{\underline{V}^{\alpha}\left(\underline{V}^{\beta}\right)^{*}: \alpha, \beta \in \tilde{\Lambda}\right\}, \psi$ is the unique such completely positive map. Now consider the minimal Stinespring dilation of $\psi$. So we have a Hilbert space $\tilde{\mathcal{H}}$ containing $\mathcal{H}$, and a unital $*$-homomorphism $\pi: C^{*}(\underline{V}) \rightarrow \mathcal{B}(\tilde{\mathcal{H}})$, such that

$$
\psi(X)=\left.P_{\mathcal{H}} \pi(X)\right|_{\mathcal{H}} \forall X \in C^{*}(\underline{V}),
$$

and $\overline{\operatorname{span}}\left\{\pi(X) h: X \in C^{*}(\underline{V}), h \in \mathcal{H}\right\}=\tilde{\mathcal{H}}$. Taking $\underline{\tilde{V}}=\left(\tilde{V}_{1}, \ldots, \tilde{V}_{n}\right)=\left(\pi\left(V_{1}\right)\right.$, $\left.\ldots, \pi\left(V_{n}\right)\right)$, one verifies that each $\left(\tilde{V}_{i}\right)^{*}$ leaves $\mathcal{H}$ invariant and $\tilde{V}$ is the unique minimal isometric dilation of $\underline{T}$.

In a similar fashion, if $\underline{T}$ is commuting, by considering $C^{*}(\underline{S})$ instead of $C^{*}(\underline{V})$, and restricting $A_{r}$ in the range to $\Gamma_{s}\left(\mathbb{C}^{n}\right)$, and taking limits as before (see [5], 24], 2]), we obtain the unique unital completely positive map $\phi: C^{*}(\underline{S}) \rightarrow \mathcal{B}(\mathcal{H})$ satisfying

$$
\phi\left(\underline{S}^{\alpha}\left(\underline{S}^{\beta}\right)^{*}\right)=\underline{T}^{\alpha}\left(\underline{T}^{\beta}\right)^{*}, \quad \alpha, \beta \in \tilde{\Lambda} .
$$

Consider the minimal Stinespring dilation of $\phi$. Here we have a Hilbert space $\mathcal{H}_{1}$ containing $\mathcal{H}$ and a unital $*$-homomorphism $\pi_{1}: C^{*}(\underline{S}) \rightarrow \mathcal{B}\left(\mathcal{H}_{1}\right)$ such that

$$
\phi(X)=\left.P_{\mathcal{H}} \pi_{1}(X)\right|_{\mathcal{H}} \forall X \in C^{*}(\underline{S}),
$$

and $\overline{\operatorname{span}}\left\{\pi_{1}(X) h: X \in C^{*}(\underline{S}), h \in \mathcal{H}\right\}=\mathcal{H}_{1}$. Taking $\underline{\tilde{S}}=\left(\tilde{S}_{1}, \ldots, \tilde{S}_{n}\right)=$ $\left(\pi_{1}\left(S_{1}\right), \ldots, \pi_{1}\left(S_{n}\right)\right)$, we see that $\underline{\tilde{S}}$ is the standard commuting dilation of $\underline{T}$ by definition (it is not difficult to verify that it is a minimal dilation in the sense of our Definition 2). As minimal Stinespring dilation is unique up to unitary equivalence, standard commuting dilation is also unique up to unitary equivalence.

Theorem 13 (Main Theorem). Suppose $\underline{T}$ is a commuting contractive tuple on a Hilbert space $\mathcal{H}$. Then the maximal commuting piece of the minimal isometric dilation of $\underline{T}$ is a realization of the standard commuting dilation of $\underline{T}$.

Our approach to proving this theorem is as follows. First we consider the standard commuting dilation of $\underline{T}$ on a Hilbert space $\mathcal{H}_{1}$ as described above. Now the standard tuple $\underline{S}$ is also a contractive tuple. So we have a unique unital completely positive map $\eta: C^{*}(\underline{V}) \rightarrow C^{*}(\underline{S})$ satisfying

$$
\eta\left(\underline{V}^{\alpha}\left(\underline{V}^{\beta}\right)^{*}\right)=\underline{S}^{\alpha}\left(\underline{S}^{\beta}\right)^{*}, \quad \alpha, \beta \in \tilde{\Lambda} .
$$

Now clearly $\psi=\phi \circ \eta$. Consider the minimal Stinespring dilation of the composed map $\pi_{1} \circ \eta: C^{*}(\underline{V}) \rightarrow \mathcal{B}\left(\mathcal{H}_{1}\right)$. Here we obtain a Hilbert space $\mathcal{H}_{2}$ containing $\mathcal{H}_{1}$ and a unital $*$-homomorphism $\pi_{2}: C^{*}(\underline{V}) \rightarrow \mathcal{B}\left(\mathcal{H}_{2}\right)$ such that

$$
\pi_{1} \circ \eta(X)=\left.P_{\mathcal{H}_{1}} \pi_{2}(X)\right|_{\mathcal{H}_{1}}, \forall X \in C^{*}(\underline{V}),
$$


and $\overline{\operatorname{span}}\left\{\pi_{2}(X) h: X \in C^{*}(\underline{V}), h \in \mathcal{H}_{1}\right\}=\mathcal{H}_{2}$. Now we have a commuting diagram as follows:

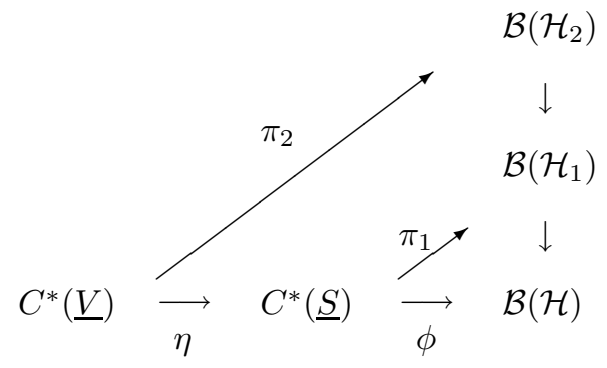

where the down arrows are compression maps, the horizontal arrows are unital completely positive maps and the diagonal arrows are unital $*$-homomorphisms.

Taking $\hat{\underline{V}}=\left(\hat{V}_{1}, \ldots, \hat{V}_{n}\right)=\left(\pi_{2}\left(V_{1}\right), \ldots, \pi_{2}\left(V_{n}\right)\right)$, we need to show that (i) $\hat{\underline{V}}$ is the minimal isometric dilation of $\underline{T}$, and (ii) $\underline{\tilde{S}}=\left(\pi_{1}\left(S_{1}\right), \ldots, \pi_{1}\left(S_{n}\right)\right)$ is the maximal commuting piece of $\hat{\underline{V}}$. Due to uniqueness up to unitary equivalence of the minimal Stinespring dilation, we have (i) if we can show that $\pi_{2}$ is a minimal dilation of $\psi=\phi \circ \eta$. For proving this we actually make use of (ii). First we prove (ii) in a very special case.

Definition 14. An $n$-tuple $\underline{T}=\left(T_{1}, \ldots, T_{n}\right)$ of operators on a Hilbert space $\mathcal{H}$ is called a spherical unitary if it is commuting, each $T_{i}$ is normal, and $T_{1} T_{1}^{*}+\cdots+$ $T_{n} T_{n}^{*}=I$.

Actually, if $\mathcal{H}$ is a finite dimensional Hilbert space and $\underline{T}$ is a commuting tuple on $\mathcal{H}$ satisfying $\sum T_{i} T_{i}^{*}=I$, then it is automatically a spherical unitary, that is, each $T_{i}$ is normal. This is the case because here a standard commuting dilation of $\underline{T}$ is a tuple of normal operators, and hence each $T_{i}^{*}$ is subnormal (or see [6] for this result), and all finite dimensional subnormal operators are normal (see [19]).

Note that if $\underline{T}$ is a spherical unitary, we have

$$
\phi\left(\underline{S}^{\alpha}\left(I-\sum S_{i} S_{i}^{*}\right)\left(\underline{S}^{\beta}\right)^{*}\right)=\underline{T}^{\alpha}\left(I-\sum T_{i} T_{i}^{*}\right)\left(\underline{T}^{\beta}\right)^{*}=0
$$

for any $\alpha, \beta \in \tilde{\Lambda}$. This forces $\phi(X)=0$ for any compact operator $X$ in $C^{*}(\underline{S})$. Now, as the commutators $\left[S_{i}^{*}, S_{j}\right]$ are all compact, we see that $\phi$ is a unital *homomorphism. So the minimal Stinespring dilation of $\phi$ is itself. So the following result yields Theorem 13 for spherical unitaries.

Theorem 15. Let $\underline{T}$ be a spherical unitary on a Hilbert space $\mathcal{H}$. Then the maximal commuting piece of the minimal isometric dilation of $\underline{T}$ is $\underline{T}$.

As proof of this theorem involves some lengthy computations, we prefer to postpone it. But assuming this, we prove the Main Theorem.

Proof of Theorem 13. As $C^{*}(\underline{S})$ contains the ideal of all compact operators, by standard $C^{*}$-algebra theory we have a direct sum decomposition of $\pi_{1}$ as follows. Take $\mathcal{H}_{1}=\mathcal{H}_{1 C} \oplus \mathcal{H}_{1 N}$, where $\mathcal{H}_{1 C}=\overline{\operatorname{span}}\left\{\pi_{1}(X) h: h \in \mathcal{H}, X \in C^{*}(\underline{S})\right.$ and $X$ 
is compact $\}$ and $\mathcal{H}_{1 N}=\mathcal{H}_{1} \ominus \mathcal{H}_{1 C}$. Clearly $\mathcal{H}_{1 C}$ is a reducing subspace for $\pi_{1}$. Therefore

$$
\pi_{1}(X)=\left(\begin{array}{ll}
\pi_{1 C}(X) & \\
& \pi_{1 N}(X)
\end{array}\right),
$$

that is, $\pi_{1}=\pi_{1 C} \oplus \pi_{1 N}$, where

$$
\pi_{1 C}(X)=P_{\mathcal{H}_{1 C}} \pi_{1}(X) P_{\mathcal{H}_{1 C}}, \quad \pi_{1 N}(X)=P_{\mathcal{H}_{1 N}} \pi_{1}(X) P_{\mathcal{H}_{1 N}} .
$$

As observed by Arveson [5], $\pi_{1 C}(X)$ is just the identity representation with some multiplicity. More precisely, $\mathcal{H}_{1 C}$ can be factored as $\mathcal{H}_{1 C}=\Gamma_{s}\left(\mathbb{C}^{n}\right) \otimes \overline{\Delta_{\underline{T}}(\mathcal{H})}$, so that $\pi_{1 C}(X)=X \otimes I$; in particular $\pi_{1 C}\left(S_{i}\right)=S_{i} \otimes I$. Also $\pi_{1 N}(X)=0$ for compact $X$. Therefore, taking $Z_{i}=\pi_{1 N}\left(S_{i}\right)$, we see that $\underline{Z}=\left(Z_{1}, \ldots, Z_{n}\right)$ is a spherical unitary.

Now, $\pi_{1} \circ \eta=\left(\pi_{1 C} \circ \eta\right) \oplus\left(\pi_{1 N} \circ \eta\right)$ and the minimal Stinespring dilation of a direct sum of two completely positive maps is the direct sum of minimal Stinespring dilations. So $\mathcal{H}_{2}$ decomposes as $\mathcal{H}_{2}=\mathcal{H}_{2 C} \oplus \mathcal{H}_{2 N}$, where $\mathcal{H}_{2 C}, \mathcal{H}_{2 N}$ are orthogonal reducing subspaces of $\pi_{2}$, such that $\pi_{2}$ also decomposes, say $\pi_{2}=\pi_{2 C} \oplus \pi_{2 N}$, with

$$
\pi_{1 C} \circ \eta(X)=\left.P_{\mathcal{H}_{1 C}} \pi_{2 C}(X)\right|_{\mathcal{H}_{1 C}}, \pi_{1 N} \circ \eta(X)=\left.P_{\mathcal{H}_{1 N}} \pi_{2 N}(X)\right|_{\mathcal{H}_{1 N}},
$$

for $X \in C^{*}(\underline{V})$ with $\mathcal{H}_{2 C}=\overline{\operatorname{span}}\left\{\pi_{2 C}(X) h: X \in C^{*}(\underline{V}), h \in \mathcal{H}_{1 C}\right\}$ and $\mathcal{H}_{2 N}=\overline{\operatorname{span}}\left\{\pi_{2 N}(X) h: X \in C^{*}(\underline{V}), h \in \mathcal{H}_{1 N}\right\}$. It is also not difficult to see that $\mathcal{H}_{2 C}=\overline{\operatorname{span}}\left\{\pi_{2 C}(X) h: X \in C^{*}(\underline{V}), X\right.$ compact, $\left.h \in \mathcal{H}_{1 C}\right\}$ and hence $\mathcal{H}_{2 C}$ factors as $\mathcal{H}_{2 C}=\Gamma\left(\mathbb{C}^{n}\right) \otimes \overline{\Delta_{\underline{T}}(\mathcal{H})}$ with $\pi_{2 C}\left(V_{i}\right)=V_{i} \otimes I$. Also $\left(\pi_{2 N}\left(V_{1}\right), \ldots, \pi_{2 N}\left(V_{n}\right)\right)$ is a minimal isometric dilation of the spherical isometry $\left(Z_{1}, \ldots, Z_{n}\right)$. Now by Proposition 6 , Theorem 15 and Corollary 5 , we get that $\left(\pi_{1}\left(S_{1}\right), \ldots, \pi_{1}\left(S_{n}\right)\right)$ acting on $\mathcal{H}_{1}$ is the maximal commuting piece of $\left(\pi_{2}\left(V_{1}\right), \ldots, \pi_{2}\left(V_{n}\right)\right)$.

All that remains to show is that $\pi_{2}$ is the minimal Stinespring dilation of $\phi \circ \eta$. Suppose this is not the case. Then we get a reducing subspace $\mathcal{H}_{20}$ for $\pi_{2}$ by taking $\mathcal{H}_{20}=\overline{\operatorname{span}}\left\{\pi_{2}(X) h: X \in C^{*}(\underline{V}), h \in \mathcal{H}\right\}$. Take $\mathcal{H}_{21}=\mathcal{H}_{2} \ominus \mathcal{H}_{20}$ and correspondingly decompose $\pi_{2}$ as $\pi_{2}=\pi_{20} \oplus \pi_{21}$,

$$
\pi_{2}(X)=\left(\begin{array}{ll}
\pi_{20}(X) & \\
& \pi_{21}(X)
\end{array}\right) .
$$

Note that we already have $\mathcal{H} \subseteq \mathcal{H}_{20}$. We claim that $\mathcal{H}_{2} \subseteq \mathcal{H}_{20}$. First, as $\mathcal{H}_{1}$ is the space where the maximal commuting piece of $\left(\pi_{2}\left(V_{1}\right), \ldots, \pi_{2}\left(V_{n}\right)\right)=\left(\pi_{20}\left(V_{1}\right) \oplus\right.$ $\left.\pi_{21}\left(V_{1}\right), \ldots, \pi_{20}\left(V_{n}\right) \oplus \pi_{21}\left(V_{n}\right)\right)$ acts, by the first part of Corollary $5, \mathcal{H}_{1}$ decomposes as $\mathcal{H}_{1}=\mathcal{H}_{10} \oplus \mathcal{H}_{11}$ for some subspaces $\mathcal{H}_{10} \subseteq \mathcal{H}_{20}$ and $\mathcal{H}_{11} \subseteq \mathcal{H}_{21}$. So, for $X \in C^{*}(\underline{V}), P_{\mathcal{H}_{1}} \pi_{2}(X) P_{\mathcal{H}_{1}}$ has the form (see the diagram)

$$
P_{\mathcal{H}_{1}} \pi_{2}(X) P_{\mathcal{H}_{1}}=\left(\begin{array}{cccc}
\pi_{10} \circ \eta(X) & 0 & & \\
0 & 0 & & \\
& & \pi_{11} \circ \eta(X) & 0 \\
& & 0 & 0
\end{array}\right),
$$

where $\pi_{10}, \pi_{11}$ are compressions of $\pi_{1}$ to $\mathcal{H}_{10}, \mathcal{H}_{11}$ respectively. As the mapping $\eta$ from $C^{*}(\underline{V})$ to $C^{*}(\underline{S})$ is clearly surjective, it follows that $\mathcal{H}_{10}, \mathcal{H}_{11}$ are reducing subspaces for $\pi_{1}$. Now as $\mathcal{H}$ is contained in $\mathcal{H}_{20}$, in view of the minimality of $\pi_{1}$ as a Stinespring dilation, $\mathcal{H}_{1} \subseteq \mathcal{H}_{20}$. But then the minimality of $\pi_{2}$ shows that $\mathcal{H}_{2} \subseteq \mathcal{H}_{20}$. Therefore, $\mathcal{H}_{2}=\mathcal{H}_{20}$.

Proof of Theorem 15. Here we need a different presentation of the minimal isometric dilation. This is known as the Schäffer construction [27] in the one-variable 
case, and 21] is a good reference for the multivariate case. Here we decompose the dilation space $\tilde{\mathcal{H}}$ as $\tilde{\mathcal{H}}=\mathcal{H} \oplus\left(\Gamma\left(\mathbb{C}^{n}\right) \otimes \mathcal{D}\right)$, where $\mathcal{D}$ is the closure of the range of the operator

$$
D: \underbrace{\mathcal{H} \oplus \cdots \oplus \mathcal{H}}_{n \text { copies }} \rightarrow \underbrace{\mathcal{H} \oplus \cdots \oplus \mathcal{H}}_{n \text { copies }}
$$

and $D$ is the positive square root of

$$
D^{2}=\left[\delta_{i j} I-T_{i}^{*} T_{j}\right]_{n \times n} .
$$

Whenever it is convenient for us, we identify $\underbrace{\mathcal{H} \oplus \cdots \oplus \mathcal{H}}_{n \text { copies }}$ with $\mathbb{C}^{n} \otimes \mathcal{H}$, so that

$$
\left(h_{1}, \ldots, h_{n}\right)=\sum_{i=1}^{n} e_{i} \otimes h_{i}
$$

Then

$$
D\left(h_{1}, \ldots, h_{n}\right)=D\left(\sum_{i=1}^{n} e_{i} \otimes h_{i}\right)=\sum_{i=1}^{n} e_{i} \otimes\left(h_{i}-\sum_{j=1}^{n} T_{i}^{*} T_{j} h_{j}\right) .
$$

And the minimal isometric dilation $\tilde{V}_{i}$ has the form

$$
\tilde{V}_{i}\left(h \oplus \sum_{\alpha \in \tilde{\Lambda}} e^{\alpha} \otimes d_{\alpha}\right)=T_{i} h \oplus D\left(e_{i} \otimes h\right) \oplus e_{i} \otimes\left(\sum_{\alpha \in \tilde{\Lambda}} e^{\alpha} \otimes d_{\alpha}\right)
$$

for $h \in \mathcal{H}, d_{\alpha} \in \mathcal{D}$ for $\alpha \in \tilde{\Lambda}$, and $1 \leq i \leq n\left(\mathbb{C}^{n} \omega \otimes \mathcal{D}\right.$ has been identified with $\left.\mathcal{D}\right)$.

In the present case, as $\sum T_{i} T_{i}^{*}=I$, by direct computation $D^{2}$ is seen to be a projection. So, $D$, which is the positive square root of $D^{2}$, is equal to $D^{2}$. Also, by the Fuglede-Putnam theorem ([19], [26]), $\left\{T_{1}, \ldots, T_{n}, T_{1}^{*}, \ldots, T_{n}^{*}\right\}$ forms a commuting family of operators. Then we get

$$
D\left(h_{1}, \ldots, h_{n}\right)=\sum_{i, j=1}^{n} e_{i} \otimes T_{j}\left(T_{j}^{*} h_{i}-T_{i}^{*} h_{j}\right)=\sum_{i, j=1}^{n} e_{i} \otimes T_{j}\left(h_{i j}\right),
$$

where $h_{i j}=T_{j}^{*} h_{i}-T_{i}^{*} h_{j}$ for $1 \leq i, j \leq n$. Note that $h_{i i}=0$ and $h_{j i}=-h_{i j}$.

Now we apply Proposition 4 to the tuple $\underline{\tilde{V}}$ acting on $\tilde{\mathcal{H}}$. Suppose

$$
y \in \mathcal{H}^{\perp} \cap \tilde{\mathcal{H}}^{c}(\underline{\tilde{V}}) .
$$

We wish to show that $y=0$. We assume $y \neq 0$ and arrive at a contradiction. One can decompose $y$ as $y=0 \oplus \sum_{\alpha \in \tilde{\Lambda}} e^{\alpha} \otimes y_{\alpha}$, with $y_{\alpha} \in \mathcal{D}$. If for some $\alpha, y_{\alpha} \neq 0$, then $\left\langle\omega \otimes y_{\alpha},\left(\tilde{\tilde{V}}^{\alpha}\right)^{*} y\right\rangle=\left\langle e^{\alpha} \otimes y_{\alpha}, y\right\rangle=\left\langle y_{\alpha}, y_{\alpha}\right\rangle \neq 0$. Since each $\left(\tilde{V}_{i}\right)^{*}$ leaves $\tilde{\mathcal{H}}^{c}(\underline{\tilde{V}})$ invariant, $\left(\underline{\tilde{V}}^{\alpha}\right)^{*} y \in \tilde{\mathcal{H}}^{c}(\underline{\tilde{V}})$. So without loss of generality we can assume $\left\|y_{0}\right\|=1$.

Taking $\tilde{y}_{m}=\sum_{\alpha \in \Lambda^{m}} e^{\alpha} \otimes y_{\alpha}$, we get $y=0 \oplus \bigoplus_{m>0}\left(\tilde{y}_{m}\right)$. As $y_{0} \in \mathcal{D}, y_{0}=$ $D\left(h_{1}, \ldots, h_{n}\right)$, for some $\left(h_{1}, \ldots, h_{n}\right)$ (presently, $D$ being a projection, its range is closed). Set $\tilde{x_{0}}=\tilde{y_{0}}=y_{0}$, and for $m \geq 1$,

$$
\tilde{x}_{m}=\sum_{i_{1}, \ldots, i_{m-1}, i, j=1}^{n} e_{i_{1}} \otimes \cdots \otimes e_{i_{m-1}} \otimes e_{i} \otimes D\left(e_{j} \otimes T_{i_{1}}^{*} \ldots T_{i_{m-1}}^{*} h_{i j}\right) .
$$


Clearly $\tilde{x}_{m} \in\left(\mathbb{C}^{n}\right)^{\otimes m} \otimes \mathcal{D}$ for all $m \in \mathbb{N}$. From the definition (3.2) of $\tilde{V}_{i}$, commutativity of the operators $T_{i}$, and the fact that $D$ is projection, we have

$$
\begin{aligned}
& \sum_{1 \leq i<j \leq n}\left(\tilde{V}_{i} \tilde{V}_{j}-\tilde{V}_{j} \tilde{V}_{i}\right) h_{i j} \\
= & \sum_{1 \leq i<j \leq n}\left(T_{i} T_{j} h_{i j}-T_{j} T_{i} h_{i j}\right)+\sum_{1 \leq i<j \leq n} D\left(e_{i} \otimes T_{j} h_{i j}-e_{j} \otimes T_{i} h_{i j}\right) \\
& +\sum_{1 \leq i<j \leq n}\left(e_{i} \otimes D\left(e_{j} \otimes h_{i j}\right)-e_{j} \otimes D\left(e_{i} \otimes h_{i j}\right)\right) \\
= & D\left\{\sum_{1 \leq i<j \leq n}\left(e_{i} \otimes T_{j} h_{i j}-e_{j} \otimes T_{i} h_{i j}\right)\right\}+\sum_{i, j=1}^{n} e_{i} \otimes D\left(e_{j} \otimes h_{i j}\right) \\
= & D\left(\sum_{i, j=1}^{n} e_{i} \otimes T_{j} h_{i j}\right)+\sum_{i, j=1}^{n} e_{i} \otimes D\left(e_{j} \otimes h_{i j}\right) \\
= & D^{2}\left(h_{1}, \ldots, h_{n}\right)+\sum_{i, j=1}^{n} e_{i} \otimes D\left(e_{j} \otimes h_{i j}\right) \\
= & \tilde{x}_{0}+\tilde{x}_{1} .
\end{aligned}
$$

Therefore $\left\langle y, \tilde{x}_{0}+\tilde{x}_{1}\right\rangle=0$ by Proposition 4 . Now for $m \geq 2$

$$
\begin{aligned}
& \sum_{i_{1}, \ldots, i_{m-1}=1}^{n} \tilde{V}_{i_{1}} \ldots \tilde{V}_{i_{m-1}}\left(\sum_{i, j=1}^{n}\left(\tilde{V}_{i} \tilde{V}_{j}-\tilde{V}_{j} \tilde{V}_{i}\right) T_{i_{1}}^{*} \ldots T_{i_{m-2}}^{*} T_{j}^{*} h_{i_{m-1} i}\right) \\
= & \sum_{i_{1}, \ldots, i_{m-1}=1}^{n} \tilde{V}_{i_{1}} \ldots \tilde{V}_{i_{m-1}}\left[\sum _ { i , j = 1 } ^ { n } D \left(e_{i} \otimes T_{j} T_{i_{1}}^{*} \ldots T_{i_{m-2}}^{*} T_{j}^{*} h_{i_{m-1} i}\right.\right. \\
+ & \sum_{i, j=1}^{n}\left\{e_{j} \otimes D\left(e_{j} \otimes T_{i} T_{i_{1}}^{*} \ldots T_{i_{m-2}}^{*} \ldots T_{j}^{*} h_{i_{m-1} i}^{*} T_{j}^{*} h_{i_{m-1} i}\right)\right. \\
= & \left.\left.\sum_{i_{1}, \ldots, i_{m-1}=1}^{n} e_{i_{1}}^{n} \otimes \ldots \otimes e_{i_{m-1}} \otimes D\left(e_{i} \otimes T_{i_{1}}^{*} \ldots T_{i_{m-2}}^{*} T_{j}^{*} h_{i_{m-1} i}\right)\right\}\right] \\
\otimes & {\left[D\left(\sum_{i, j=1}^{n} e_{i} \otimes T_{j} T_{i_{1}}^{*} \ldots T_{i_{m-2}}^{*} T_{j}^{*} h_{i_{m-1} i}-e_{j} \otimes T_{i} T_{i_{1}}^{*} \ldots T_{i_{m-2}}^{*} T_{j}^{*} h_{i_{m-1} i}\right)\right.} \\
+ & \left\{\sum_{i, j=1}^{n} e_{i} \otimes D\left(e_{j} \otimes T_{i_{1}}^{*} \ldots T_{i_{m-2}}^{*} T_{j}^{*} h_{i_{m-1} i}\right)\right. \\
& \left.\left.-\sum_{i, j=1}^{n} e_{i} \otimes D\left(e_{j} \otimes T_{i_{1}}^{*} \ldots T_{i_{m-2}}^{*} T_{i}^{*} h_{i_{m-1} j}\right)\right\}\right]
\end{aligned}
$$

(in the term above, $i$ and $j$ have been interchanged in the last summation) 


$$
\begin{aligned}
= & \sum_{i_{1}, \ldots, i_{m-1}=1}^{n} e_{i_{1}} \otimes \cdots \otimes e_{i_{m-1}} \\
& \otimes\left[D\left(\sum_{i=1}^{n} e_{i} \otimes T_{i_{1}}^{*} \ldots T_{i_{m-2}}^{*} h_{i_{m-1}}-\sum_{i, j=1}^{n} e_{j} \otimes T_{i} T_{i_{1}}^{*} \ldots T_{i_{m-2}}^{*} T_{j}^{*} h_{i_{m-1}}\right)\right. \\
& \left.+\sum_{i, j=1}^{n} e_{i} \otimes D\left\{e_{j} \otimes\left(T_{i_{1}}^{*} \ldots T_{i_{m-2}}^{*} T_{j}^{*} h_{i_{m-1} i}-T_{i_{1}}^{*} \ldots T_{i_{m-2}}^{*} T_{i}^{*} h_{i_{m-1} j}\right)\right\}\right] \\
= & \sum_{i_{1}, \ldots, i_{m-1}=1}^{n} e_{i_{1}} \otimes \cdots \otimes e_{i_{m-1}} \otimes \sum_{i=1}^{n} D\left(e_{i} \otimes T_{i_{1}}^{*} \ldots T_{i_{m-2}}^{*} h_{i_{m-1} i}\right) \\
& +\sum_{i_{1}, \ldots, i_{m-1}, i, j=1}^{n} e_{i_{1}} \otimes \cdots \otimes e_{i_{m-1}} \otimes e_{i} \\
& \otimes D\left(e_{j} \otimes T_{i_{1}}^{*} \ldots T_{i_{m-2}}^{*}\left(T_{j}^{*} T_{i}^{*} h_{i_{m-1}}-T_{j}^{*} T_{i_{m-1}}^{*} h_{i}-T_{i}^{*} T_{j}^{*} h_{i_{m-1}}+T_{i}^{*} T_{i_{m-1}}^{*} h_{j}\right)\right) \\
= & \sum_{i_{1}, \ldots, i_{m-2}, i, j=1}^{n} e_{i_{1}} \otimes \cdots \otimes e_{i_{m-2}} \otimes e_{i} \otimes D\left(e_{j} \otimes T_{i_{1}}^{*} \ldots T_{i_{m-2}}^{*} h_{i j}\right) \\
& +\sum_{i_{1}, \ldots, i_{m-1}, i, j=1}^{n} e_{i_{1}} \otimes \cdots \otimes e_{i_{m-1}} \otimes e_{i}
\end{aligned}
$$

(in the term above, index $i_{m-1}$ has been replaced by $i$ and $i$ has been replaced by $j$ in the first summation)

$$
\begin{aligned}
= & \sum_{i_{1}, \ldots, i_{m-2}, i, j=1}^{n} e_{i_{1}} \otimes \cdots \otimes e_{i_{m-2}} \otimes e_{i} \otimes D\left(e_{j} \otimes T_{i_{1}}^{*} \ldots T_{i_{m-2}}^{*} h_{i j}\right) \\
& -\sum_{\substack{i_{1}, \ldots, i_{m-1}, i, j=1 \\
\tilde{x}_{m-1}-\tilde{x}_{m} .}}^{n} e_{i_{1}} \otimes \cdots \otimes e_{i_{m-1}} \otimes e_{i} \otimes D\left(e_{j} \otimes T_{i_{1}}^{*} \ldots T_{i_{m-1}}^{*} h_{i j}\right) \\
= &
\end{aligned}
$$

So, $\left\langle y, \tilde{x}_{m-1}-\tilde{x}_{m}\right\rangle=0$.

Next, we would show that $\left\|\tilde{x}_{m+1}\right\|=\left\|\tilde{x}_{0}\right\|=1$ for all $m \in \mathbb{N}$. Indeed,

$$
\begin{gathered}
\left\|\tilde{x}_{m+1}\right\|^{2}=\left\langle\sum_{i_{1}, \ldots, i_{m}, i, j=1}^{n} e_{i_{1}} \otimes \cdots \otimes e_{i_{m}} \otimes e_{i} \otimes D\left(e_{j} \otimes T_{i_{1}}^{*} \ldots T_{i_{m}}^{*} h_{i j}\right),\right. \\
=\sum_{i_{1}, \ldots, i_{m}, i=1}^{n}\left\langle\sum_{j=1}^{n} D\left(e_{j} \otimes T_{i_{1}}^{*} \ldots T_{i_{m}}^{*} h_{i j}\right), \sum_{j^{\prime}=1}^{n} D\left(e_{j^{\prime}} \otimes T_{i_{1}}^{*} \ldots T_{i_{m}}^{*} h_{i j^{\prime}}\right)\right\rangle \\
\left.=\sum_{i_{1}, \ldots, i_{m}, i=1}^{n}\left\langle D\left(\sum_{j=1}^{n} e_{j} \otimes T_{i_{1}}^{*} \ldots T_{i_{m}}^{*} h_{i j}\right), \sum_{j^{\prime}=1}^{n} e_{j^{\prime}} \otimes T_{j^{\prime}} \otimes T_{i_{1}^{\prime}}^{*} \ldots T_{i_{m}^{\prime}}^{*} h_{i^{\prime} j^{\prime}}\right)\right\rangle
\end{gathered}
$$




$$
\begin{aligned}
& =\sum_{i_{1}, . ., i_{m}, i=1}^{n}\left\langle\sum_{l, k=1}^{n} e_{l} \otimes T_{k}\left(T_{k}^{*} T_{i_{1}}^{*} \ldots T_{i_{m}}^{*} h_{i l}-T_{l}^{*} T_{i_{1}}^{*} \ldots T_{i_{m}}^{*} h_{i k}\right),\right. \\
& \left.\sum_{j^{\prime}=1}^{n} e_{j^{\prime}} \otimes T_{i_{1}}^{*} \ldots T_{i_{m}}^{*} h_{i j^{\prime}}\right\rangle \\
& =\sum_{i_{1}, ., i_{m}, i, j=1}^{n}\left\langle\sum_{k=1}^{n} T_{k}\left(T_{k}^{*} T_{i_{1}}^{*} \ldots T_{i_{m}}^{*} h_{i j}-T_{j}^{*} T_{i_{1}}^{*} \ldots T_{i_{m}}^{*} h_{i k}\right), T_{i_{1}}^{*} \ldots T_{i_{m}}^{*} h_{i j}\right\rangle \\
& =\sum_{i, j=1}^{n}\left\langle\sum_{k=1}^{n}\left(T_{k} T_{k}^{*} h_{i j}-T_{k} T_{j}^{*} h_{i k}\right), h_{i j}\right\rangle \\
& =\sum_{i, j=1}^{n}\left\langle\sum_{k=1}^{n}\left(T_{k} T_{k}^{*} T_{j}^{*} h_{i}-T_{k} T_{k}^{*} T_{i}^{*} h_{j}-T_{k} T_{j}^{*} T_{k}^{*} h_{i}+T_{k} T_{j}^{*} T_{i}^{*} h_{k}\right), T_{j}^{*} h_{i}-T_{i}^{*} h_{j}\right\rangle \\
& =\sum_{i, j=1}^{n}\left\langle\sum_{k=1}^{n}\left(T_{k} T_{j}^{*} T_{i}^{*} h_{k}-T_{k} T_{k}^{*} T_{i}^{*} h_{j}\right), T_{j}^{*} h_{i}-T_{i}^{*} h_{j}\right\rangle \\
& =\sum_{i, j=1}^{n}\left\langle\sum_{k=1}^{n}\left(T_{k} T_{j}^{*} T_{i}^{*} h_{k}\right)-T_{i}^{*} h_{j}, T_{j}^{*} h_{i}-T_{i}^{*} h_{j}\right\rangle \\
& =\sum_{i, j=1}^{n}\left\langle T_{j}\left(\sum_{k=1}^{n} T_{k} T_{j}^{*} T_{i}^{*} h_{k}\right)-T_{j} T_{i}^{*} h_{j}, h_{i}\right\rangle \\
& -\sum_{i, j=1}^{n}\left\langle T_{i}\left(\sum_{k=1}^{n} T_{k} T_{j}^{*} T_{i}^{*} h_{k}\right)-T_{i} T_{i}^{*} h_{j}, h_{j}\right\rangle \\
& =\sum_{i=1}^{n}\left\langle\sum_{j=1}^{n} T_{j} T_{j}^{*}\left(\sum_{k=1}^{n} T_{k} T_{i}^{*} h_{k}\right)-\sum_{j=1}^{n} T_{j} T_{i}^{*} h_{j}, h_{i}\right\rangle \\
& -\sum_{j=1}^{n}\left\langle\sum_{i=1}^{n} T_{i} T_{i}^{*}\left(\sum_{k=1}^{n} T_{k} T_{j}^{*} h_{k}\right)-\sum_{i=1}^{n} T_{i} T_{i}^{*} h_{j}, h_{j}\right\rangle \\
& =\sum_{i=1}^{n}\left\langle\left(\sum_{k=1}^{n} T_{k} T_{i}^{*} h_{k}\right)-\sum_{j=1}^{n} T_{j} T_{i}^{*} h_{j}, h_{i}\right\rangle-\sum_{j=1}^{n}\left\langle\sum_{k=1}^{n} T_{k} T_{j}^{*} h_{k}-h_{j}, h_{j}\right\rangle \\
& \left.=\sum_{j=1}^{n}\left\langle h_{j}-\sum_{k=1}^{n} T_{k} T_{j}^{*} h_{k}\right), h_{j}\right\rangle=\left\langle D\left(h_{1}, \ldots, h_{n}\right),\left(h_{1}, \ldots, h_{n}\right)\right\rangle=\left\|\tilde{x}_{0}\right\|^{2}=1 .
\end{aligned}
$$

As $\left\langle y, \tilde{x}_{0}+\tilde{x}_{1}\right\rangle=0$ and $\left\langle y, \tilde{x}_{m}-\tilde{x}_{m+1}\right\rangle=0$ for $m \in \mathbb{N}$, we get $\left\langle y, \tilde{x}_{0}+\tilde{x}_{m+1}\right\rangle=0$ for $m \in \mathbb{N}$. This implies $1=\left\langle\tilde{y}_{0}, \tilde{y}_{0}\right\rangle=\left\langle\tilde{y}_{0}, \tilde{x}_{0}\right\rangle=-\left\langle\tilde{y}_{m+1}, \tilde{x}_{m+1}\right\rangle$. By the CauchySchwarz inequality, $1 \leq\left\|\tilde{y}_{m+1}\right\|\left\|\tilde{x}_{m+1}\right\|$, i.e., $1 \leq\left\|\tilde{y}_{m+1}\right\|$ for $m \in \mathbb{N}$. This is a contradiction, as $y=0 \oplus \bigoplus_{m \geq 0} \tilde{y}_{m}$ is in the Hilbert space $\tilde{\mathcal{H}}$.

\section{Representations of Cuntz algebras}

For $n \geq 2$, the Cuntz algebra $\mathcal{O}_{n}$ is the $C^{*}$-algebra generated by $n$-isometries $\underline{s}=\left\{s_{1}, \ldots, s_{n}\right\}$ satisfying the Cuntz relations: $s_{i}^{*} s_{j}=\delta_{i j} I, 1 \leq i, j \leq n$, and $\sum s_{i} s_{i}^{*}=I$. It admits many unitarily inequivalent representations. Various classes of representations of $\mathcal{O}_{n}$ have been constructed in [9], [10], [14]. Given a tuple of contractions $\underline{T}=\left(T_{1}, \ldots, T_{n}\right)$ on a Hilbert space satisfying $\sum T_{i} T_{i}^{*}=I$, we consider 
its minimal isometric dilation $\underline{\underline{V}}=\left(\tilde{V}_{1}, \ldots, \tilde{V}_{n}\right)$. We know that the isometries $\tilde{V}_{i}$ satisfy Cuntz relations, and we obtain a representation $\pi_{\underline{T}}$ of the Cuntz algebra $\mathcal{O}_{n}$ by setting $\pi_{\underline{T}}\left(s_{i}\right)=\tilde{V}_{i}$. We wish to classify all representations of $\mathcal{O}_{n}$ we can obtain by dilating commuting contractive tuples $\underline{T}$.

Let $\mathcal{S}_{n}=C\left(\partial B_{n}\right)$ be the $C^{*}$-algebra of all continuous complex valued functions on the sphere $\partial B_{n}=\left\{\left(z_{1}, \ldots, z_{n}\right): \sum\left|z_{i}\right|^{2}=1\right\}$. We have a distinguished tuple $\underline{z}=\left(z_{1}, \ldots, z_{n}\right)$ of elements in $\mathcal{S}_{n}$ consisting of co-ordinate functions. Given any spherical unitary $\underline{Z}=\left(Z_{1}, \ldots, Z_{n}\right)$ there is a unique representation of $\mathcal{S}_{n}$ which maps $z_{i}$ to $Z_{i}$. Now given any commuting $n$-tuple of operators $\underline{T}$, satisfying $\sum T_{i} T_{i}^{*}=I$, we consider its standard commuting dilation $\underline{\tilde{S}}=\left(\tilde{S}_{1}, \ldots, \tilde{S_{n}}\right)$. Let $\rho_{\underline{\underline{T}}}$ be the representation of $\mathcal{S}_{n}$ obtained by taking $\rho_{\underline{\underline{T}}}\left(z_{i}\right)=\tilde{S}_{i}$.

Definition 16. Let $\pi$ be a representation of $\mathcal{O}_{n}$ on a Hilbert space $\mathcal{L}$ with $\underline{W}=$ $\left(W_{1}, \ldots, W_{n}\right)=\left(\pi\left(s_{1}\right), \ldots, \pi\left(s_{n}\right)\right)$. The representation $\pi$ is said to be spherical if $\overline{\operatorname{span}}\left\{\underline{W^{\alpha}} h: h \in \mathcal{L}^{c}(\underline{W}), \alpha \in \tilde{\Lambda}\right\}=\mathcal{L}$, where $\mathcal{L}^{c}(\underline{W})$ is the space where the maximal commuting piece $\underline{W}^{c}$ of $\underline{W}$ acts as in Definition 3 .

Note that this definition means in particular that if $\pi$ is spherical then the maximal commuting piece $\underline{W}^{c}$ is nontrivial. We will see that it is actually a spherical unitary. But this is not a justification for calling such representations spherical, because this happens for any representation of $\mathcal{O}_{n}$, as long as $\underline{W}^{c}$ is nontrivial! The actual justification of this definition is in Theorem 18.

Theorem 17. Let $\underline{T}=\left(T_{1}, \ldots, T_{n}\right)$ be a commuting tuple of operators on a Hilbert space $\mathcal{H}$, satisfying $\sum T_{i} T_{i}^{*}=I$. Then the representation $\pi_{\underline{T}}$ coming from the minimal isometric dilation of $\underline{T}$ is spherical. Suppose $\underline{R}=\left(R_{1}, \ldots, R_{n}\right)$ is another commuting tuple, possibly on a different Hilbert space, satisfying $\sum R_{i} R_{i}^{*}=I$. Then the representations $\pi_{\underline{T}}, \pi_{\underline{R}}$ of $\mathcal{O}_{n}$ are unitarily equivalent if and only if the representations $\rho_{\underline{\underline{T}}}, \rho_{\underline{R}}$ of $\mathcal{S}_{n}$ are unitarily equivalent.

Proof. In view of Theorem 13, the maximal commuting piece of the minimal isometric dilation $\underline{\tilde{V}}$ of $\underline{T}$ is a realization of the standard commuting dilation $\underline{\tilde{S}}$ of $\underline{T}$. The first claim follows easily, as the space on which the standard commuting dilation acts includes the original space $\mathcal{H}$. So $\underline{V}$ is the minimal isometric dilation of $\underline{\tilde{S}}$. A similar statement holds for the tuple $\underline{R}$. Now the theorem follows due to the uniqueness up to equivalence of a minimal isometric dilation of contractive tuples, and the unitary equivalence of maximal commuting pieces of unitarily equivalent tuples.

So this theorem reduces the classification problem for representations of $\mathcal{O}_{n}$ arising out of general commuting tuples to that of representations of $\mathcal{S}_{n}$. But $\mathcal{S}_{n}$ being a commutative $C^{*}$-algebra, its representations are well-understood and is part of standard $C^{*}$-algebra theory. We find the description of this theory as presented in Arveson's classic [4 most suitable for our purposes.

Given any point $w=\left(w_{1}, \ldots w_{n}\right) \in \partial B_{n}$, we have a one-dimensional representation $\phi_{w}$ of $\mathcal{S}_{n}$ which maps $f$ to $f(w)$. Of course $w$ is a spherical unitary as an operator tuple on $\mathbb{C}$. We can construct the minimal isometric dilation $\left(W_{1}^{w}, \ldots, W_{n}^{w}\right)$ of this tuple as in the proof of Theorem 15 (Schäffer construction). We see that the dilation space is

$$
\mathcal{H}^{w}=\mathbb{C} \oplus\left(\Gamma\left(\mathbb{C}^{n}\right) \otimes \mathbb{C}_{w}^{n}\right) \subseteq \mathbb{C} \oplus\left(\Gamma\left(\mathbb{C}^{n}\right) \otimes \mathbb{C}^{n}\right),
$$


where $\mathbb{C}_{w}^{n}$ is the subspace of vectors orthogonal to $\left(\overline{w_{1}}, \ldots, \overline{w_{n}}\right)$ in $\mathbb{C}^{n}$. Further, the operators $W_{i}^{w}$ are given by

$$
W_{i}^{w}\left(h \oplus \sum_{\alpha} e^{\alpha} \otimes d_{\alpha}\right)=w_{i} h \oplus D\left(e_{i} \otimes h\right) \oplus e_{i} \otimes\left(\sum_{\alpha} e^{\alpha} \otimes d_{\alpha}\right) .
$$

We denote the associated representation of $\mathcal{O}_{n}$ by $\rho_{w}$. This representation is known to be irreducible, as it is nothing but the GNS representation of the so-called Cuntz state on $\mathcal{O}_{n}$ (see [14], Example 5.1), given by

$$
s_{i_{1}} \cdots s_{i_{m}} s_{j_{1}}^{*} \cdots s_{j_{p}}^{*} \mapsto w_{i_{1}} \cdots w_{i_{m}}{\overline{w_{j}}}_{1} \cdots \overline{w_{j}} .
$$

Now an arbitrary multiplicity-free representation of $\mathcal{S}_{n}$ can be described as follows (see 4]). Consider a finite Borel measure $\mu$ on $\partial B_{n}$. Then we get a representation of $\mathcal{S}_{n}$ on the Hilbert space $L^{2}\left(\partial B_{n}, \mu\right)$, which sends $f \in \mathcal{S}_{n}$ to the operator 'multiplication by $f$ '. This representation can be thought of as a direct integral of representations $\phi_{w}$ with respect to a measure $\mu$. Now it is not hard to see that the associated representation of $\mathcal{O}_{n}$ is simply the direct integral of representations $\rho_{w}$ with respect to $\mu$ and acts on $\oint \mathcal{H}^{w} \mu(d w)$. Finally, an arbitrary representation of $\mathcal{S}_{n}$ is a countable direct sum of such multiplicity-free representations. So we have proved the following result.

Theorem 18. Every spherical representation of $\mathcal{O}_{n}$ is a direct integral of representations $\rho_{w}, w \in \partial B_{n}$ (GNS representations of Cuntz states).

Here we have not bothered to say when two such representations are equivalent. But in view of Theorem 17, we can do it exactly as in (4], pages 54-55), by keeping track of multiplicities and equivalence classes of measures.

Theorem 19. Let $\pi$ be a representation of $\mathcal{O}_{n}$. Then:

(i) $\pi$ decomposes uniquely as $\pi=\pi^{0} \oplus \pi^{1}$, where $\pi^{0}$ is spherical and $\left(\pi^{1}\left(s_{1}\right), \ldots\right.$, $\left.\pi^{1}\left(s_{n}\right)\right)$ has trivial maximal commuting piece (either $\pi^{0}$ or $\pi^{1}$ could also be absent).

(ii) The maximal commuting piece of $\left(\pi\left(s_{1}\right), \ldots, \pi\left(s_{n}\right)\right)$ either is trivial or it is a spherical unitary.

(iii) If $\pi$ is irreducible, then either the maximal commuting piece is trivial or it is one-dimensional. In the second case, it is unitarily equivalent to the GNS representation of a Cuntz state.

Proof. Suppose $\pi$ is a representation of $\mathcal{O}_{n}$ on a Hilbert space $\mathcal{L}$ and

$$
\underline{W}=\left(\pi\left(s_{1}\right), \ldots, \pi\left(s_{n}\right)\right) .
$$

Consider the space $\mathcal{L}^{0}$ generated by $\mathcal{L}^{c}(\underline{W})$ as $\mathcal{L}^{0}=\overline{\operatorname{span}}\left\{\underline{W^{\alpha}} h: h \in \mathcal{L}^{c}(\underline{W}), \alpha \in\right.$ $\tilde{\Lambda}\}$. Now each $W_{i}^{*}$ leaves $\mathcal{L}^{c}(\underline{W})$ invariant, and clearly $\mathcal{O}_{n}=C^{*}\left\{\underline{s}^{\alpha}\left(\underline{s}^{\beta}\right)^{*}: \alpha, \beta \in \tilde{\Lambda}\right\}$. Then it follows that $\mathcal{L}^{0}$ is a reducing subspace for $\pi$. Taking $\mathcal{L}^{1}=\left(\mathcal{L}^{0}\right)^{\perp}$, we decompose $\pi$ as $\pi^{0} \oplus \pi^{1}$ with respect to $\mathcal{L}=\mathcal{L}^{0} \oplus \mathcal{L}^{1}$. It is clear that this is a decomposition as required by (i). Uniqueness of this decomposition and (ii) follow easily, as a maximal commuting piece of a direct sum of tuples is a direct sum of maximal commuting pieces (Corollary 5), and then (iii) follows from Theorem 18.

Let us see what happens if we dilate commuting tuples $\underline{T}$ satisfying just $\sum T_{i} T_{i}^{*} \leq$ I. In this case, as is well-known, the minimal isometric dilation decomposes as $\left(\left(V_{1} \otimes I\right) \oplus W_{1}, \ldots,\left(V_{n} \otimes I\right) \oplus W_{n}\right)$, where $\left(V_{1}, \ldots, V_{n}\right)$ is the standard tuple of full Fock space, and $\left(W_{1}, \ldots, W_{n}\right)$ are isometries satisfying Cuntz relations. If $\underline{T}$ is not 
pure, the term $\left(W_{1}, \ldots, W_{n}\right)$ is present and we get a representation of $\mathcal{O}_{n}$. However, as seen in the proof of Theorem $13,\left(W_{1}, \ldots, W_{n}\right)$ is a minimal isometric dilation of a spherical tuple $\left(Z_{1}, \ldots, Z_{n}\right)$ (the 'spherical part' of the standard commuting dilation of $\underline{T}$ ), and hence the representation of $\mathcal{O}_{n}$ we get is still spherical.

On the other hand, it is easy to get examples of noncommuting tuples dilating to representations of $\mathcal{O}_{n}$ which are not spherical. For instance, we can consider the tuple $\underline{R}=\left(R_{1}, R_{2}\right)$ on $\mathbb{C}^{2}$ defined by

$$
R_{1}=\left[\begin{array}{ll}
0 & 1 \\
0 & 0
\end{array}\right], \quad R_{2}=\left[\begin{array}{ll}
0 & 0 \\
1 & 0
\end{array}\right] .
$$

Then as $R_{1} R_{1}^{*}+R_{2} R_{2}^{*}=I$, the minimal isometric dilation of $\left(R_{1}, R_{2}\right)$ satisfies the Cuntz relations. We can see that it has trivial commuting piece through a simple application of Corollary 4.3 of [14].

Finally, we remark that if we are to consider the case $n=\infty$, that is, if we have infinite tuples $\left\{T_{1}, T_{2}, \ldots,\right\}$, then the standard commuting tuple $\left\{S_{1}, S_{2}, \ldots,\right\}$ no longer consists of essentially normal operators, as the commutators $\left[S_{i}^{*}, S_{i}\right]$ have infinite dimensional eigenspaces with nonzero eigenvalues. This is a serious obstacle in extending the results of Sections 3 and 4 to infinite tuples. Taking a different direction, many results have been now extended to the case of $q$-commuting tuples $\left(T_{j} T_{i}=q_{i j} T_{i} T_{j}\right.$ with $\left.q_{i j} \in \mathbb{C}\right)$ by S. Dey in 15 .

\section{ACKNOWLEDGEMENTS}

The first author is supported by Indo-French (IFCPAR) grant No. IFC/\#01$1 / 2001 / 1253$, the second author is supported by research grant no. SR/FTP/MS16/2001 of the Department of Science and Technology, India, and the last author is supported by a research fellowship from the Indian Statistical Institute.

\section{REFERENCES}

[1] J. Agler, 'The Arveson extension theorem and coanalytic models', Integral Equations and Operator Theory, 5 (1982) 608-631. MR 84g:47011

[2] A. Arias and G. Popescu, 'Noncommutative interpolation and Poisson transforms,' Israel J. Math., 115 (2000) 205-234. MR 2001i:47021

[3] A. Arias and G. Popescu, 'Noncommutative interpolation and Poisson transforms II', Houston J. Math., 25 (1999) 79-98. MR 2000i:47148

[4] W. B. Arveson, An Invitation to $C^{*}$-algebras, Graduate Texts in Mathematics, No. 39, Springer-Verlag, New York-Heidelberg, 1976. MR 58:23621

[5] W. B. Arveson, 'Subalgebras of $C^{*}$-algebras III, Multivariable operator theory,' Acta Math., 181 (1998) 159-228. MR 2000e:47013

[6] A. Athavale, 'On the intertwining of joint isometries,' J. Operator Theory, 23 (1990) 339-350. MR 91i:47029

[7] A. Athavale, 'Model theory on the unit ball in $\mathbb{C}^{m}$, J. Operator Theory, 27 (1992) 347-358. MR 94i:47011

[8] B. V. Rajarama Bhat and T. Bhattacharyya, 'A model theory for $q$-commuting contractive tuples', J. Operator Theory 47 (2002) 97-116. MR 2003c:47018

[9] O. Bratteli and Palle E. T. Jorgensen Iterated function systems and permutation representations of the Cuntz algebra, Mem. Amer. Math. Soc., 139 (1999), no. 663. MR 99k:46094a

[10] O. Bratteli and Palle E. T. Jorgensen, 'Isometries, shifts, Cuntz algebras and multiresolution wavelet analysis of scale $N$ ', Integral Equations Operator Theory, 28 (1997) 382-443. MR 99k:46094b

[11] J. W. Bunce, 'Models for $n$-tuples of noncommuting operators,' J. Funct. Anal., 57 (1984) 21-30. MR 85k:47019 
[12] J. Cuntz, 'Simple $C^{*}$-algebras generated by isometries,' Commun. Math. Phys., 57 (1977) 173-185. MR 57:7189

[13] C. Davis, 'Some dilation and representation theorems', Proceedings of the Second International Symposium in West Africa on Functional Analysis and its Applications (Kumasi, 1979), Forum for Funct. Anal. Appl., Kumasi, Ghana, 1979, pp. 159-182. MR 84e:47012

[14] K. R. Davidson, D. W. Kribs, and M. E. Shpigel, 'Isometric dilations of non-commuting finite rank $n$-tuples', Canad. J. Math., 53 (2001) 506-545. MR 2002f:47010

[15] Dey, S. 'Standard dilations of $q$-commuting tuples', Indian Statistical Institute, Bangalore preprint (2003)

[16] S. W. Drury, 'A generalization of von Neumann's inequality to the complex ball', Proc. Amer. Math. Soc., 68 (1978) 300-304. MR 80c: 47010

[17] A. E. Frazho, 'Models for noncommuting operators', J. Funct. Anal., 48 (1982) 1-11. MR 84h:47010

[18] A. E. Frazho, 'Complements to models for noncommuting operators', J. Funct. Anal., 59 (1984) 445-461. MR 86h:47010

[19] P. R. Halmos, A Hilbert Space Problem Book, Second Edition, Graduate Texts in Mathematics, No. 19, Springer-Verlag, New York-Berlin, 1982. MR 84e:47001.

[20] S. Parrott, 'Unitary dilations for commuting contractions', Pacific J. Math.. 34 (1970) 481490. MR 42:3607

[21] G. Popescu, 'Isometric dilations for infinite sequences of noncommuting operators', Trans. Amer. Math. Soc., 316 (1989) 523-536. MR 90c:47006

[22] G. Popescu, 'Models for infinite sequences of noncommuting operators', Acta Sci. Math. (Szeged), 53 (1989) 355-368. MR 91b:47025

[23] G. Popescu, 'Characteristic functions for infinite sequences of noncommuting operators', $J$. Operator Theory, 22 (1989) 51-71. MR 91m:47012

[24] G. Popescu, 'Poisson transforms on some $C^{*}$-algebras generated by isometries', J. Funct. Anal., 161 (1999) 27-61. MR 2000m:46117

[25] G. Popescu, 'Curvature invariant for Hilbert modules over free semigroup algebras', Advances in Mathematics, 158 (2001) 264-309. MR 2002b:46097

[26] C. R. Putnam, Commutation properties of Hilbert space Operators and Related Topics, Springer-Verlag, New York, 1967. MR 36:707

[27] J. J. Schäffer, 'On unitary dilations of contractions', Proc. Amer. Math. Soc., 6 (1955) 322. MR 16:934c

[28] B. Sz.-Nagy, C. Foias, Harmonic Analysis of Operators on Hilbert Space, North-Holland, Amsterdam (1970). MR 43:947

Indian Statistical Institute, R. V. College Post, Bangalore 560059, India

E-mail address: bhat@isibang.ac.in

Department of Mathematics, Indian Institute of Science, Bangalore 560012, India

E-mail address: tirtha@math.iisc.ernet.in

Indian Statistical Institute, R. V. College Post, Bangalore 560059, India

E-mail address: santanu@isibang.ac.in 